eigentliche Thema wird in den einzelnen Texten aber leider häufig gar nicht eingegangen. Es findet sich zusammengewürfeltes Wissen mit durchaus bedenkenswerten Details wie dem Hinweis auf die teilweise funktionale Repräsentation in Hongkongs Erster Kammer, dem Legislativen Rat, oder die ein Mal pro Jahr draußen stattfindenden Sitzungen des Tynwalds auf der Isle of Man, auf der alle im Jahr zuvor verabschiedeten Gesetze offiziell verkündet werden. Es bleibt den Lesern vorbehalten, Wesentliches zusammenzutragen und sinnvoll zusammenzuführen. So kann der Band den selbst gesetzten Anspruch, „problem charting and erecting scaffoldings for future theory-building“ (S. 17), nur bedingt einlösen.

Franziska Carstensen

\title{
Auf dem Weg zur europäischen Parteiendemokratie: wie weit ist es noch?
}

Poguntke, Thomas, Martin Morlok und Heike Merten (Hrsg.): Auf dem Weg zu einer europäischen Parteiendemokratie (Schriften zum Parteienrecht und zur Parteienforschung, Band 44), Nomos Verlagsgesellschaft, Baden-Baden 2013, 226 Seiten, € 44,-.

Das supranationale Projekt „Europäische Union“ (EU) ist bekanntermaßen nach dem Zweiten Weltkrieg als Antwort auf den Umgang mit Deutschland, den Kalten Krieg und vor allem als Friedensunion initiiert worden. Ein direkt vom „Volk“ gewähltes Parlament gibt es dabei erst seit 1979. Die Weiterentwicklung dieser Institution ist vor allem als PRAktion zu verstehen. Bis zu diesem Zeitpunkt gab es kein stichhaltiges Argument, das den Gedanken eines Elitenprojekts „EU“ hätte widerlegen können. Doch das Parlament und mehr noch seine Akteure, die Parteien, sind „auf europäischer Ebene unabdingbar für die Verwirklichung des Demokratieprinzips“ (S. 7). Die Hauptkritik nach 30 Jahren ist jedoch weiterhin, dass die nationalen Parteien den Ton angeben und dass die Wahlverfahren in den Mitgliedstaaten nicht einheitlich sind. Die Liste ließe sich um viele kleine Kritikpunkte erweitern, offensichtlich ist jedoch, dass von einer gesamteuropäischen Parteiendemokratie keine Rede sein kann. Daher muss gefragt werden, wie weit es noch bis zu dieser ist, beziehungsweise ob es überhaupt vorwärts geht.

Ausgehend von einem Symposium des Instituts für Deutsches und Internationales Parteienrecht und Parteienforschung am 20. und 21. April 2012 an der Heinrich-Heine-Universität Düsseldorf beschäftigt sich der vorliegende Band mit dem Stand und der Entwicklung der europäischen Parteiendemokratie. Die Leitfragen spiegeln dabei das vorhandene Missverhältnis zwischen, in ausreichender Zahl vorhandenen Parteien und nicht als solcher zu erkennender europäischer Parteienpolitik wider: „(1) Ist eine europäische Parteipolitik im Sinne von supranationaler Parteienregierung überhaupt denkbar? Und wenn ja: (2) Welche konstruktiven Veränderungen am europäischen Institutionengefüge und seiner Rechtsordnung sind vorzunehmen, um diese zu ermöglichen?"“ (S. 11).

Antworten hierauf werden zunächst theoretisch, dann rechtlich, im Prozess ihrer Entwicklung und schließlich nach vorn schauend angegangen. Den Anfang machen Thomas Poguntke und Hans H. Klein mit einem Überblick und Überlegungen zu Parteien in der Europäischen Union. 
Martin Morlok, Heike Merten und Gábor Motika widmen sich in jeweils eigenen Beiträgen der Parteienverordnung der EU. Durch den Bezug auf die gleichen Rechtsakte entsteht fast zwangsläufig eine gewisse Redundanz. Den Autoren gelingt es dennoch, die Quellen für ihre individuellen Themen „sprechen“ zu lassen. Eine wichtige Erkenntnis dieses Abschnitts besteht darin, dass die bisher gültige Parteienverordnung zwar lediglich eine „Parteienfinanzierungsverordnung“ ist, aber damit einen wichtigen Grundstein für die Weiterentwicklung - über den Zwischenschritt des Entwurfs von 2012 - hin zu einem europäischen Parteienstatut darstellen kann (Heike Merten, S. 66).

Wojciech Gagatek, Steven Van Hecke und Benjamin von dem Berge steuern drei Artikel zu den „Europarteien im politischen Prozess“ bei. Der letzte Beitrag sticht heraus, weil es ihm gelingt, empirisch nachzuweisen, dass eine Form der europäischen Integration auch in der Beziehung der großen Parteienverbünde EVP (Europäische Volkspartei) und SPE (Sozialdemokratische Partei Europas) zu ihren mittel- und osteuropäischen Partnerparteien zu beobachten war und ist (Benjamin von dem Berge, S. 129 - 165).

Im vierten Abschnitt beschäftigen sich Jo Leinen, Steven Van Hecke und Karl Magnus Johansson mit den „Komplexe[n] Interaktion[en] der Europarteien“. Hier steht ein Plädoyer eines Mitgliedes des Europäischen Parlaments an erster Stelle, denn gerade unter den derzeitigen Kontextbedingungen ist es notwendig, dass die Bürger verstehen, was auf der europäischen Ebene passiert und letztlich auch Chancen zur Partizipation bekommen. „Die Herausforderung, die EU zu demokratisieren, ist mindestens genauso akut wie die Lösung der Schuldenkrise im Euro-Raum" (Jo Leinen, S. 174).

Am Ende schauen Jürgen Mittag und Hans Herbert von Arnim in zwei Beiträgen auf die „Europäisierung der Europawahl“. Wichtig erscheint hier die Feststellung, dass eine europäische Parteiendemokratie zum einen nicht umgehend entstehen wird - auch nicht mit einem Parteienstatut - und zum anderen, dass mit einer gemeinsamen nationenübergreifenden Kandidatenkür ein Schritt in die richtige Richtung gegangen würde. Zusammen mit einer „schrittweisen Vereinheitlichung der Wahlverfahren“ scheint dies die nächste Wegmarke zu sein (Jürgen Mittag, S. 214).

Bedauerlicherweise wurde bei der Buchgestaltung auf Zwischentitel im Fließtext verzichtet. Darüber hinaus fällt auf, dass die zwölf Beiträge von sehr unterschiedlichem Umfang sind. Diese Differenz und die Tatsache, dass zum Teil Redemanuskripte ausführlichen wissenschaftlichen Ausarbeitungen gegenüberstehen, erzeugt eine „Unwucht“ in der Publikation.

Abschließend kann dem Band aber attestiert werden, dass er den Stand der Parteiendemokratie mit einer Vorausschau auf die Europawahl 2014 sehr gut bilanziert. Der weitere Weg ist bei der bisherigen Entwicklungsgeschwindigkeit nur schwer absehbar, trotzdem gelingt es den Autoren, die Fortschritte zu Beginn des 21. Jahrhunderts deutlich herauszustellen. Insgesamt handelt es sich um eine ergiebige Lektüre, deren kleine Redundanzen auch dem Fachneuling oder Fachfremden helfen, sich ein geschichtsträchtiges und auch zukünftig relevantes Forschungsfeld zu erschließen. 